\title{
Editorial
}

\author{
Thierry Lulle
}

\section{¡Veinte años!}

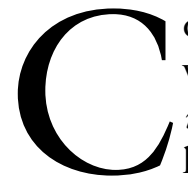

on la entrega de este número 40, tenemos el inmenso placer de celebrar los veinte años de nuestra revista. La creación de Territorios revista de estudios urbanos y regionales en 1998 deriva de la conformación de la Asociación Colombiana de Investigadores Urbano Regionales ACIUR cinco años antes (es decir, que por su lado la ACIUR - https://aciur.net/- está celebrando sus veinticinco años y, además, acaba de realizar su decimotercer seminario internacional en Barranquilla). El papel de Luis Mauricio Cuervo, uno de los fundadores de la ACIUR y director del Centro Interdisciplinario de Estudios sobre Desarrollo (CIDER) de la Universidad de los Andes en ese entonces, ha sido clave. En efecto, por tener esta doble función, él pudo facilitar la concretización de una idea que se había manifestado muy temprano en la asociación: la apertura de espacios donde la comunidad científica iba a poder presentar, difundir, compartir, discutir y debatir los resultados de sus investigaciones en torno a las dinámicas urbanas y regionales, lo cual iba a permitir reunir no solo a investigadores colombianos y extranjeros, latinoamericanos o latinoamericanistas de otras partes, sino también a actores del desarrollo urbano, regional y territorial, del sector público o de las organizaciones no gubernamentales y sociales. Estos espacios iban a ser seminarios y foros; la revista también era uno de estos espacios de reunión $\mathrm{y}$, al igual que los eventos, tenía que vivir gracias a una alianza entre la ACIUR y una universidad. Así nació la revista, Luis Mauricio mismo lo relatará muy detallada y amistosamente más adelante.

La alianza con el CIDER de la Universidad de los Andes duró diez años ${ }^{1}$, hasta que, por varias razones (salida de Luis Mauricio para la CEPAL en Chile, luego nueva orientación en los intereses del CIDER), se dio un cambio: Daniel Gómez, investigador del CIDER y vicepresidente de la ACIUR, pasó a la Universidad del Rosario y se "llevó" la revista. La transición no fue fácil, duró tres años en los que se publicó anualmente un número doble, el 16-17 en 2007, el 18-19 en 2008 y el 20-21 en 2009; sin embargo,

territarios 40 9 
${ }^{2}$ Hago parte de sus primeros miembros, soy docente investigador de la Universidad Externado de Colombia y no tengo vínculo laboral con el Rosario. En la medida en que la ACIUR tiene afiliados institucionales (una docena de universidades) a cambio del pago de una cuota anual, mi actividad de dirección de la revista equivale a la afiliación de la Universidad Externado; así mismo el papel del Rosario en la "fabricación" de la revista equivale a su afiliación a la ACIUR.

\section{territarias 40}

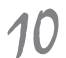

se pudo mantener la revista y esta reencontró su ritmo normal al tiempo que la editorial del Rosario se consolidaba para apoyar a la publicación de sus revistas, antiguas o más recientes, en campos muy diversos. Es con el número 18-19 que empecé a asumir la dirección de la revista, con la camisa de la ACIUR ${ }^{2}$. A pesar de esta configuración bastante especial que hubiera podido dificultar la comunicación y gestión del proceso editorial, la revista ha sido muy bien acogida por la editorial, pues ha respetado la dinámica propia de la revista, nunca ha interferido en ello, lo cual valoramos y agradecemos.

Si bien, por motivos económicos, la editorial del Rosario cambió el papel de la revista, mantuvo el formato inicial de esta. A su creación, la revista se caracterizaba por un formato específico (cuadrado), una diagramación de doble columna, y también algunas ilustraciones solicitadas a un grafista. Permaneció este formato hasta hoy, salvo las ilustraciones. Por otro lado, cada uno de los primeros números publicados con la Universidad de los Andes tenía una carátula distinta, luego se adoptó una misma carátula para todos los números hasta que, a partir del número 32, en respuesta a una solicitud del comité editorial de la revista, se empezaron a diferenciar las carátulas con un color distinto, sin alterar el formato y el diseño.

El comité editorial se conformó desde el primer número y el científico, con el número 22. En ambos casos contamos con colegas cercanos a la ACIUR y/o a las universidades con las cuales la asociación se alió, también con investigadores conocidos por nosotros de larga data o en eventos académicos y cuya especialidad podía ser de gran utilidad para el desarrollo de la revista. Entre todos ellos, se reunieron varias disciplinas y se evidenció el interés de la revista en la pluri- e interdisciplinareidad. Son colombianos o extranjeros (casi todos lo son en el comité científico), algunos se han mantenido desde el principio, otros se han ido y han llegado nuevos. Por esta composición, es evidente que, tal como lo resalta Luis Mauricio, nunca los comités fueron “endogámicos", reutilizando el término de Publindex-Colciencias al evaluar el nuestro, todo lo contrario... Por otro lado, es doloroso recordar a uno de los miembros del comité editorial, Hernán Henao, brillante investigador de la Universidad de Antioquia y uno de los fundadores de la ACIUR, a quien el conflicto, que ha sido muy duro para el país y la academia, nos quitó demasiado temprano en circunstancias trágicas.

A todos nuestros colegas, muchas gracias por haber acompañado o seguir acompañando a la revista. Aquí quisiera nombrar también a las personas que han trabajado directamente en el proceso editorial de la revista, pues el apoyo de ellas ha sido muy determinante. En primer lugar, a Clemencia Peña y a Claudia Carreño, quienes desde el CIDER acompañaron fuertemente a la revista durante su primera etapa; luego, en el Rosario, a Ana Sofía Alvarado (quien muy tristemente falleció mientras estaba trabajando con mucho ánimo durante la etapa de transición); también a Jaime Prada, Camilo A. Suárez y Sarah Nieto, coordinadora editorial desde hace cinco años, todos(as) 
ellos(as) han trabajado al tiempo para Territorios y la revista Desafios que publica la Facultad de Ciencia Política, Gobierno y Relaciones Internacionales de la Universidad del Rosario. Y todo el equipo de la editorial del Rosario: Juan Felipe Córdoba, su director, con quien se trabaja desde la llegada de la revista al Rosario, Diego Esteban Romero, María José Molano y Claudia Amparo Luque, actual editora de publicaciones periódicas; contribuyeron a la consolidación de la organización editorial, así como al cumplimiento de las normas y criterios de indexación. A todos(as) ellos(as) muchísimas gracias por su compromiso y constantes y valiosos aportes.

El número de artículos recibidos no deja de crecer. Actualmente, entre el $20 \%$ y el $30 \%$ de ellos son aprobados por los pares evaluadores, a menudo con recomendaciones de ajustes. Es bastante común la autoría colectiva y se destaca el hecho de que nuestros autores son tanto hombres como mujeres. Tanto los autores como los pares evaluadores no son solo de Colombia, sino también de otros países latinoamericanos, Europa y Estados Unidos. La gran mayoría de los pares siempre han tenido un alto nivel académico, algunos nos acompañan desde hace mucho tiempo, otros de manera más puntual porque los solicitamos en un campo muy específico. Todos han contribuido a la calidad de la revista. Para un director de revista esta etapa de la identificación de los pares evaluadores no es siempre una tarea fácil, pero sabemos que tampoco para los pares mismos. También con ellos nos sentimos muy agradecidos.

Los artículos se publican en castellano y a veces en portugués, hasta ahora ninguno en inglés. Se puede observar la presencia de varias disciplinas: si las dominantes son la arquitectura, la planeación urbana, la economía urbana o el derecho, la aparición de temas sociales y culturales procede desde otras disciplinas (sociología, antropología, psicología social, trabajo social, historia, geografía, etc.); por otro lado, se puede constatar, tal como la revista lo ha buscado desde su creación, la colaboración entre autores de distintas disciplinas. Por otra parte, vale la pena resaltar la diversidad de la naturaleza de los proyectos, cuyos resultados se presentan en los artículos en la medida en que determina el tipo de datos, procesamiento y análisis: las investigaciones de carácter científico (a veces son tesis doctorales); las consultorías financiadas por el Estado u ONG; los análisis de datos estadísticos producidos por los institutos nacionales de estadísticas o por gremios del sector inmobiliario o finca raíz; experiencias de intervención social o en el mundo profesional; tesis de pre y posgrado. Por último, hay que señalar una tensión entre lo teórico-conceptual y lo empírico, los estudios de casos, etc. Se ve cómo el peso de los paradigmas de las ciencias sociales -materialismo histórico, (post-)estructuralismo e incluso la emergencia de nuevos paradigmas venidos de las teorías de la complejidad o del caos)- poco a poco se fueron debilitando para dejar más espacio a trabajos empíricos. Obviamente, en este proceso se gana y 
pierde a la vez, pero siempre se trata de seguir propiciando una sólida construcción de conocimientos.

Por otro lado, a la editorial le tocó manejar todo el proceso de indexación que se ha vuelto cada vez más pesado. Como lo recuerda Luis Mauricio, desde sus inicios la revista procede a la evaluación de los artículos por pares, lo cual en ese momento no era muy común, pero también opta por el diálogo de saberes, invitando al debate entre investigadores y otros actores del desarrollo urbano, regional y territorial. Poco después (Luis Mauricio menciona con mucha precisión el n. ${ }^{\circ} 11$ de 2004 , es decir bien antes del paso de la revista del CIDER al Rosario), Publindex-Colciencias empezó el proceso de indexación, el cual ha sido "traumatizante", Luis Mauricio habla de "castigo" pues, mientras la revista tenía al lado de una sección de artículos científicos otra sección que permitía que actores del desarrollo urbano y regional (sector público, organizaciones sociales) pudieran presentar su reflexión sobre sus experiencias, por efectos de la aplicación de ciertos criterios de indexación por Publindex se tuvo que suspender dicha sección con mucha decepción. Ahora bien, sin someterse ciegamente a estas reglas discutibles y a veces aplicadas erradamente (por ejemplo, varias veces Publindex ha considerado que de la composición de nuestro comité científico no era suficientemente internacional cuando, al revés, casi todos sus miembros son extranjeros desde su creación), poco a poco la revista trató de cumplir con ellas teniendo en cuenta expectativas de autores cuyo reconocimiento puede pasar por la publicación en revistas indexadas, de tal suerte que de hecho la revista pudo volver a ser indexada por Publindex-Colciencias. Por otro lado, la revista se incorporó progresivamente a otras plataformas de indexación (hoy son dieciséis); una ha sido muy importante, la Redalyc, en la medida en que se trata de una red latinoamericana y caribeña que trató de diferenciarse de las redes anglosajonas y norteamericanas. Actualmente, la revista está a la espera de su incorporación a Scopus. Y, si bien no pudo subir en la última clasificación de Publindex, cuyo modelo de medición ha cambiado fuertemente no sin generar mucha controversia, no bajó su posición, lo cual ha sido un "éxito" dado la gran cantidad de revistas que no pudieron mantenerse en su categoría.

Desde el primer número, la revista tiene una sección temática, pero es a partir del número 25 que se formaliza la colaboración con editores invitados; ellos se responsabilizan de dicha sección temática. Muchos de ellos, colombianos y extranjeros, son expertos muy reconocidos en el tema para el cual los hemos invitado. También pudo haber números con artículos que derivaron de ponencias presentadas en eventos de la ACIUR o de una red parecida como fue el caso de la Red Latinoamericana de Teoría Urbana (RELATEUR -http://www.relateur.org/-), sin nunca haber dejado de lado la evaluación por pares de cada uno de los artículos recibidos. La colaboración de estos editores es muy importante para la revista y agradecemos a todos muy especialmente 
aquí. Hay que señalar también la aparición de otras dos secciones: la de "Jóvenes investigadores" y muy recientemente la de "Traducciones". La primera permite que recién graduados, tanto de un pregrado como de un posgrado, cuya tesis ha sido muy bien valorada, puedan acceder al proceso editorial; tuvimos en esta sección excelentes contribuciones. La sección "Traducciones" nos permite hacer circular trabajos muy diversos conocidos en otros ámbitos de otro idioma, mas no en los nuestros. La sección general, por su lado, siempre se ha mantenido.

Vale la pena revisar ahora los temas abordados en la revista, pues de alguna manera evidencian también cómo la investigación urbana, regional y territorial se ha configurado a lo largo de los últimos veinte años. Nos referimos a los temas de las secciones temáticas -no los abordados en las otras secciones- que, si bien no concuerdan siempre en el tiempo, son muy vecinos a los de los seminarios de la ACIUR, en especial los siguientes:

- Desarrollo: Desarrollo local (n. ${ }^{\circ}$ 1, agosto 1998-enero 1999); Desarrollo económico territorial (n. ${ }^{\circ} 20-21$, enero-diciembre 2009), es interesante resaltar que precisamente fue el tema del primer número.

- Políticas públicas: Ciudad y políticas urbanas (n. ${ }^{\circ}$ 13, agosto 2004-enero 2005); Balance de las políticas públicas de desarrollo territorial (n. ${ }^{\circ} 24$, enero-junio 2011 ).

- Gobierno: Democracia, descentralización y desarrollo (n. ${ }^{\circ} 5$, agosto 2000 -enero 2001); Gobernabilidad local (n. ${ }^{\circ} 8$, febrero-julio 2002); Gobierno de municipios y aglomeraciones urbanas (I) $\left(n{ }^{\circ}{ }^{2} 2\right.$, enero-junio 2010$)$ y (II) (n. ${ }^{\circ} 23$, julio-diciembre 2010).

- Mercados: Mercados inmobiliarios populares (n. ${ }^{\circ}$ 18-19, enero-diciembre 2008); Reconfiguración territorial y mercados de tierras rurales ( $n .^{\circ} 30$ enero-junio 2014).

- Servicios: Ciudad: servicios y ordenamiento (n. ${ }^{\circ}$ 14, febrero-julio 2005); Movilidad cotidiana y transporte (.$^{\circ} 25$, julio-diciembre 2011$)$.

- Aspectos sociales y políticos: Construcción social de la ciudad (n. ${ }^{\circ} 6$, febrero-julio 2001 ); Construcción social del territorio (n. ${ }^{\circ} 10-11$, febrero 2003 -enero 2004); Marginalidad y territorio ( $\mathrm{n} .^{\circ}$ 9, agosto 2002-enero 2003); Género, desarrollo y territorio (n. ${ }^{\circ}$ 16-17, enero-diciembre 2007); Violencia urbana (n. ${ }^{\circ} 4$, febrerojulio 2000); Violencia y territorio (n. ${ }^{\circ} 15$, agosto 2005-diciembre 2006); Acción colectiva en las ciudades latinoamericanas (n. ${ }^{\circ} 31$, julio-diciembre 2014); Ciudades y conflictos en América latina: ayer, hoy y mañana I y II (n. ${ }^{\circ} 36$, enero-junio 2017 y n. ${ }^{\circ} 37$, julio-diciembre 2017); Dinámicas sociales y reconfiguraciones territoriales contemporáneas (n. ${ }^{\circ} 38$, enero-junio 2018); La diversidad de la securización urbana: dimensiones, actores y retos (n. ${ }^{\circ} 39$, julio-diciembre 2018). 
${ }^{3}$ Retomo en lo que sigue partes del capitulo que escribimos Angélica Camargo, Peter Brand (actualy anterior presidentes de la ACIUR) y yo mismo, titulado "La investigación y la formación en lo urbano regionalen Colombia:entre avances marcados y grandes retos”, el cual se publicó en Pascale Metzger, Julien Rebotier, Jérémy Robert, Patricia, Urquieta es Pablo Vega-Centeno (editores científicos) (2016). La cuestión urbana en la región andina: miradas sobre la investigación y la formación (pp. 77-115), Quito: PUCE, IRD, IFEA.

\section{territarias 40}

- Ambiental: Desastres (n. ${ }^{\circ} 3$, agosto 1999-enero 2000); Territorios, naturaleza y medio ambiente (n. ${ }^{\circ} 26$, enero-junio 2012); Ciudades y resiliencia: riesgo, vulnerabilidad y adaptación en América Latina (n. ${ }^{\circ} 28$, enero-junio 2013).

- Historia urbana: Procesos de ocupación del territorio, historia urbana y patrimonio (I) (n. ${ }^{\circ} 32$, enero-junio 2015$)$ y (II) (n. ${ }^{\circ} 33$, julio-diciembre 2015$)$.

- Teoría: Repensar las ciudades contemporáneas (n. ${ }^{\circ} 7$, agosto 2001 -enero 2002); Prismas de pensamiento ( . $^{\circ}$ 12, febrero-julio 2004); Estado del arte de la investigación urbano-regional en América Latina (n. ${ }^{\circ}$ 27, julio-diciembre 2012); La vigencia de Henri Lefebvre en la investigación socio-territorial (n. ${ }^{\circ} 29$, juliodiciembre 2013); Teoría urbana latinoamericana I y II (n. ${ }^{\circ} 34$, enero-junio 2016 y n. ${ }^{\circ} 35$, julio-diciembre 2016$)$.

Como se puede constatar, todos los temas han sido abordados al menos dos veces y desde distintas perspectivas. En cambio, puede sorprender que algunos que han sido constantes e incluso predominantes en los seminarios de la ACIUR no hayan estado más presentes en las secciones temáticas de la revista, como en el caso del hábitat popular, tema que sí ha sido trabajado bastante en artículos de secciones generales. Hasta ahora, aunque se han planteado en el comité editorial, hay temas que todavía no han sido escogidos: por ejemplo, el multi- e interculturalismo, las tensiones entre centralidades y periferias, el impacto de las TIC (Tecnologías de Información y Comunicación) y el big data no solo en la planeación y gestión del desarrollo territorial sino también en las practicas espaciales de los ciudadanos, el posconflicto, los derechos a la vivienda, la ciudad y sus atributos, el espacio público, aunque todos estos aspectos sí fueron abordados en artículos en las distintas secciones de varios números. Si bien, tienen cierto peso la economía y la ciencia política, se observa la presencia de otras disciplinas como la geografía, la sociología, la antropología, así como la arquitectura o la planeación urbana. Finalmente, hay que señalar que mientras los artículos sobre metrópolis, ciudades grandes, son numerosos, poco a poco aparecieron artículos sobre ciudades intermedias no solo en Colombia (por ejemplo, Popayán, Tunja, Cúcuta) sino en otros países.

Podemos constatar que estos temas son bastante afines con los que se han trabajado en los seminarios de la ACIUR ${ }^{3}$. En efecto, entre los temas dominantes y constantes de dichos seminarios encontramos los siguientes:

- Desarrollo urbano regional: En este campo se han presentado investigaciones relacionadas con el crecimiento urbano y en general con las transformaciones urbanas. Desde el 2003 se contrasta con las políticas públicas de desarrollo regional y urbano. 
- Medio ambiente y sostenibilidad: El tema ambiental ha estado presente en casi todos los seminarios. Se han presentado investigaciones relacionadas con el desarrollo urbano regional, el ordenamiento territorial ambiental (probablemente debido al papel de la Ley 388/97 de Desarrollo Territorial) y, en algunos casos, sobre conflictos ambientales en relación con el desarrollo urbano, los macro-proyectos extractivos y energéticos, los efectos de los TLC, los riesgos, la vulnerabilidad ante desastres naturales y las formas de resiliencia. Por otro lado, han tenido un lugar importante aspectos propios de la gestión ambiental y procesos socioculturales o de participación en torno a lo ambiental. En los eventos más recientes han sido predominantes los temas relacionados con la sostenibilidad urbana y las preocupaciones propias del cambio climático, la gobernanza del agua, la seguridad alimentaria, el impacto de la mercantilización de la naturaleza, así como las nuevas ruralidades, los bordes urbano-rurales y el paisaje en contexto de metropolización. Por tanto, se constatan bastantes afinidades entre las preocupaciones expresadas en los seminarios y en la revista.

- Globalización y sus efectos a distintos niveles territoriales: En este espacio se han discutido fundamentalmente cuestiones de economía territorial, la mayoría de las investigaciones han abordado las relaciones entre la economía local y las economías globales. En las versiones más recientes se han abordado temas relacionados con economía urbana y espacial, en algunos casos desde el punto de vista inmobiliario. Se considera la rearticulación económica de las distintas regiones.

- Metropolización y el fenómeno de región urbana: La discusión sobre los temas metropolitanos y regionales ha estado muy presente en la investigación urbana de los últimos años, en general, se han abordado temas relacionados con el gobierno, la gestión y la institucionalidad metropolitana, la localización de actividades y las características propias (espaciales y sociopoblacionales) del proceso de metropolización.

- Gobierno urbano y participación ciudadana: Los temas relacionados con la gobernabilidad urbana, la gestión pública, la gestión presupuestaria y los procesos de descentralización y democratización han sido predominantes. Son cada vez más analizados a la luz de la organización político-administrativa (redes de poder, democracia representativa y democracia participativa), proyectos de distintas naturalezas (regeneración, renovación o revitalización urbana, recuperación del río Bogotá, protección de ecosistemas como los humedales, integración del transporte colectivo, lucha contra la inseguridad) o escalas (intra-urbana, metropolitana, regional), a veces con comparaciones internacionales, pero generalmente específicas. Poco a poco, se ha pasado a temas relacionados con la gobernanza territorial. 
- Ordenamiento territorial: En algunos casos, los temas de ordenamiento territorial se han convocado en relación con el urbanismo, la planeación urbana. Ha sido un tema importante, particularmente luego de la formulación de la Ley 388 de 1997 o Ley de Desarrollo Territorial. En este marco, se han presentado investigaciones que analizan algunos planes de ordenamiento o procesos de su formulación. Al igual que el anterior, este tema ha estado estrechamente vinculado con el derecho urbano, la evaluación del desempeño y la aplicación de instrumentos de gestión del suelo, recuperación de plusvalías y otros instrumentos de financiación del desarrollo urbano.

- Hábitat, acceso al suelo, mercado y política de vivienda: Los temas relacionados con el hábitat han estado presentes en todas las versiones del seminario de investigación con discusiones muy nutridas. En términos generales, las investigaciones han abordado temáticas relacionadas con la vivienda informal, funcionamiento de los mercados de suelo y vivienda tanto formales como informales (en este caso, la generación de una oferta de vivienda en alquiler debido a la consolidación de los barrios de origen informal), análisis de programas y políticas de vivienda social (en especial el caso de los llamados "macro-proyectos" de vivienda). Aspectos relacionados con la habitabilidad, los efectos de la "financiarización”, el derecho a la ciudad y una reflexión renovada sobre las relaciones entre la formalidad y la informalidad han ocupado un lugar importante en las últimas versiones.

- Segregación socioespacial y dinámicas de poblamiento: Esta temática ha convocado investigaciones relacionadas con las reconfiguraciones socioespaciales de las metrópolis, las dinámicas sociodemográficas (las distintas formas de movilidad espaciales, ya no solo las migraciones internas sino también las internacionales, la movilidad residencial intra-urbana/metropolitana, la movilidad cotidiana, tanto voluntarias como forzadas), aspectos relacionados con la fragmentación urbana como los efectos de la proliferación de shopping malls y conjuntos cerrados. Igualmente, el fenómeno de la gentrificación en los centros frente a las dinámicas en las periferias en un contexto general de expansión/densificación.

- Si bien, la historia urbana está presente desde los primeros eventos, la lectura histórica de los procesos de urbanización, la patrimonialización (a veces relacionada con el turismo o, como lo acabamos de señalar, la gentrificación) se ha fortalecido en los últimos eventos. Las cuestiones referentes a la (re-)configuración de las centralidades y los efectos de los proyectos de renovación urbana han aparecido en las últimas versiones.

- En algunos seminarios han tomado fuerza temas relacionados con el espacio público, con respecto a sus usos por distintos grupos sociales (dependiendo del género, la generación, lo étnico-racial, la actividad, en especial el caso de los vendedores 
ambulantes, todavía poco los discapacitados, etc.) así como a otros aspectos como la planeación, las normas vigentes en torno al espacio público, etc.

- Entre los temas menos presentes está el de servicios públicos, aunque ha sido muy importante anteriormente no solo con respecto a las prácticas populares de acceso a estos, de luchas urbanas, sino también a las políticas públicas y a la gestión de las empresas con una tendencia a la privatización. Y el transporte, aunque con el tiempo fueron tomando alguna fuerza los estudios sobre movilidad cotidiana y en algunos casos las prácticas socioespaciales (en relación con el tema de espacio público que acaba de ser evocado), los efectos de los sistemas de distintos modos de transporte sobre la vida ciudadana individual y colectiva, y las desigualdades sociales en el acceso y uso de los modos de transporte.

- En algunos de los seminarios aparecen intentos de temas nuevos, presentados durante un solo evento: por ejemplo, en el campo de la inclusión social los de género, gestión de la diversidad (en especial sexual), cuerpo, culturas ciudadanas, lo mismo que temas relacionados con la seguridad o salud; el tema "estética y ciudad" estuvo presente en el 2000 pero no continuó. Al considerar los eventos en su conjunto, se observa cómo el tema "territorial" ha venido afirmándose, y más recientemente los de "paisaje" o de "lugar".

- Finalmente, el tema bastante propio de Colombia en torno al conflicto y la paz tuvo varias expresiones dependiendo del periodo. Estuvo presente a finales de los años 2000 pero bajo la formulación de "violencia" antes de pasar a "inseguridad" la cual abarca un conjunto de situaciones más diversas (desde el conflicto armado hasta la delincuencia común urbana).

Esta doble revisión nos permite evidenciar, sin sorpresa por supuesto, bastantes cruces, lo que significa que la revista está en permanente resonancia con la investigación urbana y regional latinoamericana, la cual tiene su propio desarrollo. Es decir, que la revista asume plenamente su papel de acompañamiento en las búsquedas de los investigadores.

Hay que señalar también que en estos mismos veinte años otras revistas se han esforzado en trabajar en los mismos campos de lo urbano y territorial. Las vemos como aliadas más que competidoras, pues les toca a todas las revistas académicas enfrentar y superar las presiones de la indexación y sus consecuencias en los procesos editoriales. Pensamos en especial en Bitácora Urbano-Territorial de la Facultad de Artes de la Universidad Nacional de Colombia (desde 1997 -http://www.revistas.unal.edu.co/ index.php/bitacora-) y en los Cuadernos de Vivienda y Urbanismo del Instituto Javeriano de Vivienda y Urbanismo (Injaviu) de la Facultad de Arquitectura y Diseño de la Pontificia Universidad Javeriana (PU)) (desde 2008 -http://revistas.javeriana.edu. 
co/index.php/cvyu/index-). En todos los casos, los esfuerzos son muy importantes para lograr un buen nivel no solo en los contenidos de los artículos sino también en la presentación formal.

¿Hacia dónde vamos? Sin lugar a dudas, se presentan cada vez más retos con el paso de una lógica de la impresión a la de la difusión electrónica. Para Territorios, un primer paso importante se hizo con la apertura de la plataforma Open Journal Systems (OJS) (con ello todos los números de la revista son accesibles gratuitamente y se garantiza cierta transparencia en los procedimientos de entrega de artículos, evaluación, etc.). Probablemente, también el hecho de estar presente en las redes sociales (Facebook, Academia.edu, Google + y el perfil en Google Scholar). Sin embargo, indudablemente queda todavía mucho por hacer en este proceso de la digitalización.

\section{El número 40}

Este es un número "miscelánea", situación que nunca se presentó en la revista; sin embargo, algunos subtemas se destacan, los cuales son bastante representativos de la permanencia, reformulación y emergencia de tendencias en nuestros campos y responden de alguna manera a lo comentado anteriormente. Los primeros tres artículos se ubican en un subtema que podríamos denominar Gestión territorial y medio ambiente, el cual no ha dejado de ampliarse en la revista. En los tres casos, los autores manifiestan su preocupación por contribuir a una formulación de políticas públicas más participativas, por lo cual recurren a enfoques metodológicos pertinentes. En el artículo "La gobernanza ambiental: el estudio del capital social en las Áreas Naturales Protegidas (México)" Erika Cruz Coria, Lilia Zizumbo Villarreal y Nuchnudee Chaisatit Chaisatit, constatan que, al igual que en la mayoría de los países centroamericanos y caribeños, en México el Decreto de áreas naturales protegidas (ANP) ha surgido básicamente como un instrumento de conservación propuesta por la política de Estado; por tanto, las áreas se encuentran administradas por diversos organismos gubernamentales que limitan la participación de otros actores sociales y el aprovechamiento sustentable de los recursos en estos territorios. Frente a esta situación, desde la perspectiva teórica del capital social (CS), los autores proponen algunas herramientas teórico-metodológicas para la comprensión de la estructura de las relaciones sociales en las ANP mexicanas, y para el diseño de intervenciones planificadas encaminadas al desarrollo de formas de gobernanza ambiental. Por su parte, Tomás Canevari, Guillermo Banzato y Gastón Cirio presentan en "Percepciones de vecinos sobre problemas sociales y ambientales en el Gran La Plata, Argentina. Hacia una agenda de Gestión Integral del Territorio" resultados de una encuesta realizada en dos amplias zonas del Gran La Plata, Buenos Aires, Argentina, con posterioridad a la trágica inundación del 2 de abril de 2013. Los 
autores analizan las percepciones que los habitantes de estos barrios tenían con respecto a los problemas sociales y ambientales para aplicar los resultados en el diseño de agendas científicas de intervención y transformación. Se apoyan en el enfoque territorial de la teoría social crítica del espacio y se aplica parcialmente el Método Territorii, que aporta una visión integral de los territorios incorporando la participación de actores comunitarios, políticos, económicos y científico-técnicos. Finalmente, en el artículo "Manejo de la avifauna como parte de la gestión del arbolado urbano en Bogotá, D. C." Germán Tovar se centra en cómo el proceso de urbanización de Bogotá tiene directamente impacto en la cobertura arbórea, debido a que los proyectos de construcción priorizan la tala en vez del traslado, y sus proyectos paisajísticos compensan insuficientemente su impacto negativo. Es un tema muy poco abordado pero que se vuelve cada vez más relevante. El autor considera que la reducción progresiva de espacios verdes y arbolado disminuye los hábitats necesarios para las aves. Ahora bien, en la gestión del arbolado urbano, que incluye actividades de plantación, poda, traslado y tala, la ciudad aplica procedimientos para reducir su impacto sobre la avifauna. A partir de varias fuentes oficiales, se formulan criterios técnicos para la conectividad ecológica con énfasis en la conservación de aves y la consolidación de procesos de restauración en la Estructura Ecológica Principal de la ciudad.

Un segundo tema concierne la expansión urbana, el cual es más "tradicional" pero que se aborda ahora desde distintos enfoques. En el artículo "Crecimiento urbano y mercado de tierras en Bogotá, 1914-1944", Luis Carlos Colón se centra en el crecimiento de Bogotá en la primera mitad del siglo Xx. Revisa cuál fue el papel de los terratenientes, urbanizadores, agentes financieros y entidades municipales encargadas de la planeación en la creación y consolidación de un mercado de tierras destinado principalmente a la construcción de vivienda. El estudio considera que la acumulación de capital fue la fuerza orientadora del crecimiento urbano en este periodo, con importantes consecuencias para la ciudad en términos de los patrones de uso de la tierra, la distribución de la población y la estructura del espacio urbano en general. El autor recurre a fuentes interesantes: las escrituras notariales de los barrios inaugurados en el periodo de estudio. En el siguiente artículo "El borde no es como lo pintan. El caso del borde sur de Bogotá, D. C.”, Leonardo Garavito y Amparo de Urbina se enfocan en problematizar y evidenciar la polisemia subyacente tras la idea del borde aplicado a la ciudad y en especial al sur de Bogotá. En este sentido, los autores plantean que hay muchos y diversos bordes interactuando y superpuestos entre sí, apuntando hacia la construcción de una visión más compleja y comprensiva de este territorio.

Destacamos un tercer tema que ha estado muy presente en la investigación urbana latinoamericana desde hace varias décadas, se trata de las Politicas de vivienda. En el artículo "Arreglos neocorporatistas en la política habitacional. Un análisis de los 
Macroproyectos de Interés Social Nacional en Colombia" Óscar A. Alfonso R. plantea que el Estado contemporáneo tiene varias facetas. La del neocorporatismo es una forma moderna de representación de intereses que, en el caso de la política habitacional en Colombia, tomó cuerpo en un arreglo para producir suelo edificable con proyectos habitacionales para hogares pobres que enfrentó el impasse de la autonomía local en el ordenamiento territorial. El autor muestra cómo el arreglo neocorporatista de los Macroproyectos de Interés Social Nacional se produjo para enfrentar la crisis recesiva del sector constructor ocurrida en la coyuntura de 2008. En el artículo "Política de vivienda y gestión de suelo urbano. Casos de Corea, Colombia y Perú (1990-2015)" Julio Calderón Cockburn presenta un sugestivo análisis comparativo de los casos de Colombia, Perú y Corea del Sur, en el cual se relaciona el rol del Estado con el mercado y resalta la importancia de la gestión del suelo urbano, los programas de vivienda y la reducción de la informalidad urbana. El autor concluye que, aunque esos países han atravesado por procesos de liberalización y privatización desde la década de 1990, difieren en su esquema institucional de atención a la vivienda y gestión del suelo: un Estado desarrollador y planificador que promueve asociaciones público/ privada (Corea del Sur), otro que combina mecanismos de subsidio con gestión del suelo (Colombia) y otro en que impera el libre mercado (Perú). Finalmente, Carla Fainstein y Ariel Matías Palombi abordan en "Pragmatismo, desaliento de expectativas y gobernabilidad. La política de villas de las gestiones de Mauricio Macri en la Ciudad Autónoma de Buenos Aires (2007-2015)" las dos primeras gestiones del partido Propuesta Republicana en la Ciudad de Buenos Aires (2007-2015), las cuales supusieron una serie de novedades y continuidades en las políticas adoptadas para las villas y asentamientos de este distrito. Los autores ponen el énfasis en cómo el gobierno porteño fue transformando su perspectiva de abordaje de estas problemáticas, lo cual se vio fuertemente articulado, en consecuencia, con un creciente interés por la presencia territorial -tanto desde las instituciones estatales como de las redes políticas- en estos barrios. Por otro lado, resaltan la importancia que la judicialización de los conflictos villeros tuvo en este periodo.

Un cuarto tema menos abordado hasta el momento es el del Conflicto y acceso a la vivienda. En "El desplazamiento forzado intra-urbano: una modalidad de movilidad residencial con restricciones controvertidas (Soacha)" Tiphaine Duriez recuerda cómo, durante casi siete décadas, Colombia ha sido atravesada por diversas formas de reivindicaciones y enfrentamientos armados. Cuenta actualmente con más de 7,7 millones de desplazados internos según la ACNUR. Desde 1997, el gobierno colombiano ha adoptado una serie de medidas políticas para compensar a quienes representan

territarias 40 20 a la mayoría de sus víctimas civiles de la guerra: los desplazados forzados. La autora propone examinar los nexos existentes entre la ciudad y la guerra para presentar las 
reconfiguraciones urbanas del desplazamiento forzado a partir de observaciones de tipo etnográfico de situaciones dentro de las Comunas 4 y 6 de Soacha.

En la Sección Jóvenes investigadores, Juan David Echeverry Tamayo presenta el artículo "Medellín una ciudad de encrucijadas: pobreza, modelo de ciudad y cambio social en el proyecto Cinturón Verde". El autor señala que Medellín es una ciudad que durante años ha cargado un conjunto de problemáticas tales como unos escandalosos índices de pobreza, marginalidad y violencia, debido a la debilidad del Estado en amplias zonas territoriales. A pesar de este difícil panorama, la ciudad ha realizado esfuerzos que, si bien limitados, le han permitido ser considerada como un ejemplo de transformación urbana y social dentro de América Latina. De este modo, un megaproyecto como el Cinturón Verde ha permitido la expansión de la ciudad hacia zonas de alto riesgo para la vida humana y el ambiente.

En la Sección Traducciones presentamos el artículo “Territorios de paz: otras territorialidades en la Comunidad de Paz de San José de Apartadó, Colombia", inicialmente publicado en inglés por Christopher Courtheyn. El autor explora la territorialidad relacional generada por la "comunidad de paz" campesina en San José de Apartadó, Colombia. Partiendo del seguimiento al sujeto político colectivo producido por la activa generación de paz de la Comunidad de Paz mediante un conjunto de prácticas de espacios, lugares y valores, incluyendo conmemoraciones de masacres, iniciativas de soberanía alimentaria y redes de solidaridad entre indígenas y campesinos, este artículo presenta un marco conceptual para analizar diversas formaciones territoriales. 